\title{
All-Fibered High-Quality Low Duty-Cycle 20-GHz and 40-GHz Picosecond Pulse Sources
}

\author{
C. Finot, J. Fatome, S. Pitois and G. Millot
}

\begin{abstract}
In this work, we demonstrate all-fibered $20-\mathbf{G H z}$ and $40-\mathrm{GHz}$ picosecond pulse sources with duty cycles as low as $1 / 14$. The pulse train is achieved via the high-quality compression of an initial sinusoidal beating through four segments of optical fibers. General design rules are proposed and experimental results are in agreement with numerical predictions.
\end{abstract}

Index Terms - Pulse compression, Nonlinear optics in fibers, pulse propagation and solitons, Parabolic pulses.

\section{INTRODUCTION}

U eneration of very low duty-cycle high-quality optical pulse trains at repetition rate of tens of gigahertz around $1.5 \mu \mathrm{m}$ has become of great interest for many scientific applications such as optical sampling or ultrahigh capacity transmission systems based on Optical Time Division Multiplexing (OTDM). Unfortunately, the current bandwidth limitations of optoelectronic devices do not allow the direct generation of optical pulses having a temporal width below a few picoseconds. Nethertheless, actively mode-locked fiber lasers were found to be an efficient way to overcome this limitation, although they still remain an onerous option. As an alternative issue to conventional mode-locking operation, several techniques have been suggested, such as the conversion of a sinusoidal beating into a train of well-defined, pedestal free pulses. For instance, the use of dispersion decreasing fibers [1], step-like or comb-like fiber set-ups [2, 3], or Raman amplification [1] have been successfully shown to be all-optical approaches to generate such high repetition rate pulse trains. Recently, a technique based on multiple fourwave mixing has also demonstrated its efficiency for the generation of optical pulse trains with repetition rates ranging from $20 \mathrm{GHz}$ to $1 \mathrm{THz}$ [4]. However, those different schemes have the common feature to generate pulse trains with a high duty cycle (around 1/5). Shorter pulses are clearly feasible, but at the cost of the experimental set-up complexity [3], or with the development of noticeable substructures and pedestals in the recompressed pulses [5].

In this paper, we propose a new, passive and cost-effective approach allowing the generation of picosecond optical pulse trains with very low duty-cycle (around 1/15). The letter is organized as follow; we will first describe the physical

Manuscript received May 18, 2007.

C. Finot, J. Fatome, S. Pitois and G. Millot are with the Institut Carnot de Bourgogne (ICB), Dept. OMR, UMR CNRS 5209 Université de Bourgogne, 21078 Dijon, France. (e-mail: christophe.finot@u-bourgogne.fr). principles of our approach relying on the progressive reshaping of a sinusoidal beating in a set of carefully-chosen optical fibers. We will detail design rules enabling the rapid scaling and determination of the different elements of the setup. We will then present experimental results validating our technique at a repetition rate of $20 \mathrm{GHz}$. Other experimental data obtained at $40 \mathrm{GHz}$ will demonstrate the possibility to further extend the concept to higher repetition rates.

\section{PRINCIPLE AND DESIGN RULES OF THE CHAIN}

Our original method is based on the temporal reshaping of a sinusoidal beating in a succession of optical fibers with lengths, dispersive and non-linear properties carefully chosen. The physical evolution of the beating can be simply decomposed into four stages (illustrated in Fig. 1), each having a clear physical meaning. Our technique is thus physically distinct from set-ups relying on step-like fiber, comb-like fiber [2] or comb-like profiled fiber [3] where the succession of numerous segments mimics a dispersion decreasing fiber.

For each stage of the set-up, we have derived some semiempirical rules to optimize and facilitate the design of our pulse train compressor. Both previously published and novel analytical formula will be presented, each one taking into account the second order dispersion $\beta_{2, k}$ and the non-linear coefficient $\gamma_{k}$ of the $k^{\text {th }}$ fiber.

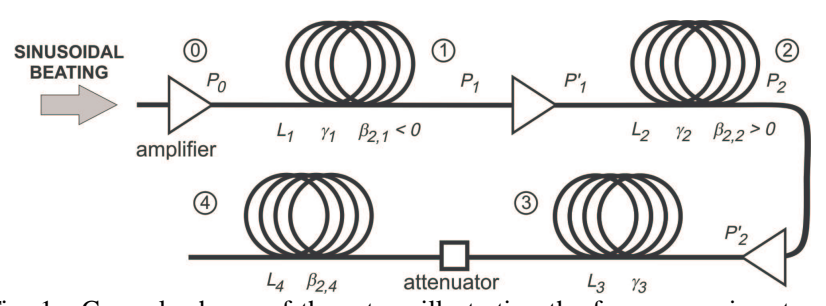

Fig. 1. General scheme of the set-up illustrating the four successive stages and their relevant parameters.

The first stage of our set-up aims to convert an initial sinusoidal beating into a well-separated Gaussian-like pulse train with a duty-cycle of $1 / 5$ through the multiple four-wave mixing process taking place into a standard anomalous fiber [4]. For a pulse train with a repetition rate $f$, it has been shown that the optimal average power $P_{0}$ at the input of the first segment is given by $P_{0} \approx 12.8\left|\beta_{2,1}\right| f^{2} / \gamma_{1}$ [4]. The optimal fiber length is $\mathrm{L}_{1} \approx 0.071 /\left|\beta_{2,1}\right| f^{2}$, providing Gaussian chirpfree pulses with negligible pedestals at the output of the first stage. The full-width at half maximum (FWHM) of the reshaped pulses is then $T_{1} \approx 1 / 5 f$ while their peak power is evaluated by $P_{1} \approx 4.57 P_{0}$. 
The second stage allows the passive reshaping of Gaussian pulses into a train of linearly chirped parabolic pulses thanks to the combined effects of self-phase modulation and normal dispersion [6]. In order to have a convenient parabolic reshaping, the optimum fiber length $L_{2}$ of the normally dispersive segment and the optimum peak power $P_{1}$ ' are $L_{2} \approx 0.006 / \beta_{2,2} f^{2}$ and $\mathrm{P}_{1}^{\prime} \approx 468 \beta_{2,2} f^{2} / \gamma_{2}$ respectively.

After reshaping inside the $L_{2}$ segment, we obtain a parabolic pulse shape having a FWHM $T_{2}$ and a peak power $P_{2}$. The pulse is linearly chirped with chirp coefficient $C_{2}$ defined as the slope of the temporal chirp. The parameters of the pulses after the second stage are then given by $T_{2} \approx 0.39 / f$, $P_{2} \approx 0.56 P^{\prime}{ }_{1}$ and $C_{2} \approx 17.3 f^{2}$.

The purpose of the third stage is essentially to increase the linear temporal chirp of the parabolic pulses in order to enhance the final recompression process. In this aim, those pulses are launched into a highly nonlinear fiber (HNLF) where they are essentially affected by self-phase modulation without suffering any temporal broadening $\left(T_{2}=T_{3}\right)$. Due to their parabolic intensity profile, the temporal chirp induced in this third segment is strictly linear [7] and for an initial peak power $P_{2}$ ' and a fiber length $L_{3}$, the amount of additional linear chirp is given by $C_{3} \approx 4.19 P_{2}^{\prime} L_{3} \quad \gamma_{3} f^{2} \quad$ [8].

In this context, at the output of the third segment, the linear chirp of the parabolic pulses $\left(C_{2}+C_{3}\right)$ can therefore be efficiently compensated by means of a usual dispersive element. A compact solution for this compensation could be a chirped fiber Bragg grating but we choose here to implement another solution by simply adding an adequate length of anomalous fiber, which constitutes the final stage of the set-up. Recompressed pulses are finally transformed-limited with a shape close to a first order Bessel function of the first kind [8] and with a temporal FWHM given by $T_{4} \approx 1.85 \mathrm{f} /\left(C_{2}+C_{3}\right)$.

Ensuring a sufficiently low power at the input of this last segment to avoid non-linear effect, the length of fiber $L_{4}$ required to achieve such a recompression is thus given by $L_{4} \approx 1 /\left[2 \pi\left(C_{2}+C_{3}\right) \beta_{2,4}\right]$.

\section{EXPERIMENTAL RESULTS AT $20 \mathrm{GHZ}$}

We have tested our approach by generating a pulse train at a repetition rate of $20 \mathrm{GHz}$. Based on the design rules described in the above section, we have settled our experimental set-up by means of commercially available devices ready for a telecom use.

The initial beating is obtained by the temporal superposition of two continuous laser diodes frequency separated by $20 \mathrm{GHz}$ (external cavity laser, ECL). A phase modulator is also inserted in order to suppress the Brillouin backscattering effect. We would like to emphasize that the generation of such a beating is fully compatible with the performances of electro-optic modulators and electronic clocks commonly used in telecommunications.

The average power of the initial beating launched into the first $8385-\mathrm{m}$ long section made of standard SMF is only 21 $\mathrm{dBm}$ [5]. The second stage is made of non-zero dispersion shifted fiber (NZ-DSF, dispersion $D=-1.5 \mathrm{ps} \cdot \mathrm{km}^{-1} \cdot \mathrm{nm}^{-1}$, $\gamma=1.7 \mathrm{~W}^{-1} \cdot \mathrm{km}^{-1}$ ) with normal dispersion whose parameters
$\left(L_{2}=7700 \mathrm{~m}, \beta_{2,2}\right)$ have been carefully chosen to match the properties of the pulses generated along the SMF and thus satisfying the relation $P_{1}=P^{\prime}{ }_{1}$. The third segment is a $500-\mathrm{m}$ long HNLF $\left(\gamma_{3}=13 \mathrm{~W}^{-1} \cdot \mathrm{km}^{-1}\right)$ with nearly zero dispersion (a slight normal dispersion is used to prevent instabilities or temporal recompression). In order to achieve a significant spectral broadening and a sufficient linear temporal chirp, we have inserted an erbium doper fiber amplifier (EDFA) at the input of the HNLF so that to reach $P_{2}^{\prime}=23 \mathrm{dBm}$. The final stage is based on a 630-m long SMF segment, preceded by an optical attenuator to ensure a linear pulse recompression without soliton recompression effect.

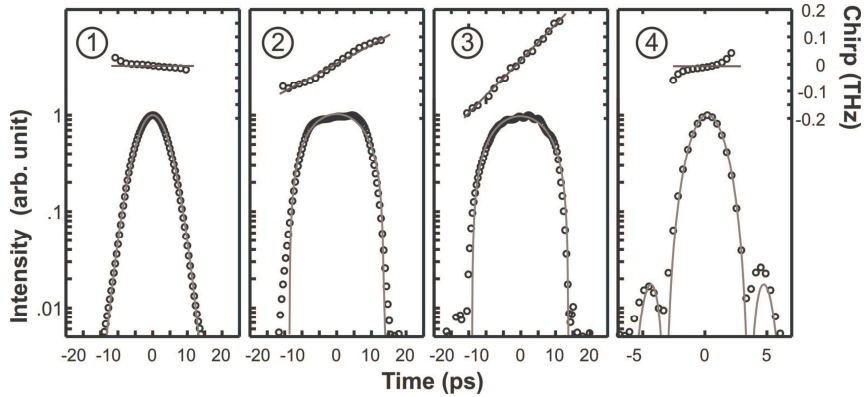

Fig. 2. Evolution of the intensity and chirp profiles (bottom and top respectively) at different stages of the set-up. 20-GHz experimental results obtained by FROG characterisation (circles) are compared to various fits (solid lines): stage 1: gaussian fit of the intensity profile, stage 2 and 3 , parabolic fits, stage 4 : fit by a Bessel function of the first kind [8]. Chirp profiles are compared to linear fits. Note that results at stage 4 are plotted with a different temporal scale than results at other stages.

We have carefully characterised both in phase and intensity the pulses at the different stages of our set-up by use of a Frequency-Resolved Optical Gating (FROG, based on second harmonic generation [9]). Experimental results are represented in Fig. 2 (circles) and are also compared to various fits corresponding to the theoretical expected shapes described in part II (solid lines). After the first stage, Fig. 2(1), we can check that the initial sinusoidal beating is efficiently compressed into a transform limited Gaussian pulse train with a duty-cycle close to $1 / 5$ (FWHM $=11$ ps and Time-Bandwith Product $=0.43$ ). In the course of the second stage, Fig. 2(2), we clearly verify that those pulses have then acquired an intensity profile in good agreement with a parabolic fit. This parabolic shape is then maintained during the whole propagation in the HNLF whereas the linear chirp is further increased (stage 3, Fig. 2(3). Finally, after recompression along the SMF segment (stage 4), the output pulses, Fig. 2(4), are nearly Fourier transformed-limited with a temporal duration of 3 ps (i.e. dutycycle of 1/16) and notably exhibit only low pedestals which are characteristic of recompressed parabolic pulses [8] (peakto-pedestal ratio larger than $17 \mathrm{~dB}$ ). Those generated pulses are then fully suitable for $80-\mathrm{GHz}$ OTDM telecommunications.

\section{EXPERIMENTAL RESULTS AT $40 \mathrm{GHZ}$}

We now present experimental results obtained at a higher repetition rate. Using the design guidelines described in part II, it is clear that higher the repetition rate $f$ is, shorter should be the length of the fibers and higher should be the peak powers launched into the different fiber segments. In other words, 
increasing the repetition rate by a factor 2 will globally lead to decrease the lengths of the segments $L_{1}$ and $L_{2}$ by a factor 4 and increase by $6 \mathrm{~dB}$ the required input power. One inherent beneficial aspect is that the power available at the output of the second fiber will be sufficiently high to observe a clear spectral broadening in the HNLF without any additional amplification, thus meaning a simplified experimental set-up made of a single line of 4 spliced optical fibers whose principal features are detailed in Fig. 3 for the $40-\mathrm{GHz}$ configuration.

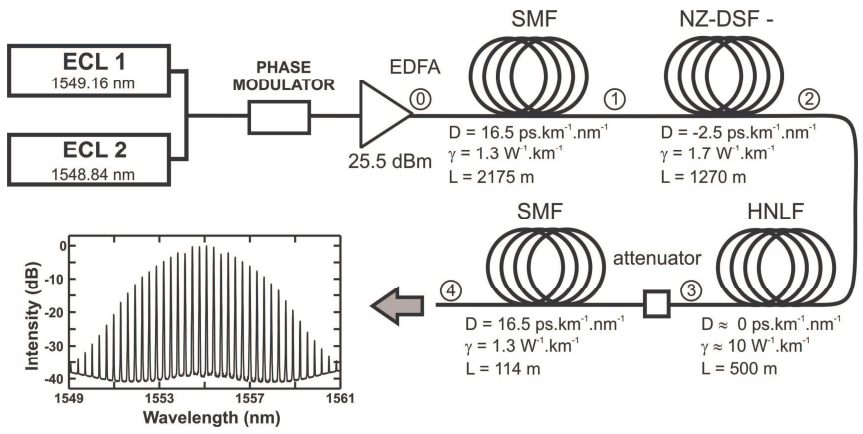

Fig. 3. 40-GHz experimental set-up, parameters and experimental optical spectrum of the $40-\mathrm{GHz}$ recompressed pulses.

We have again characterized the pulses at stages 1, 3 and 4 by means of FROG measurements. In order to fairly observe the compression process, results are plotted (Fig. 4, circles) with a temporal window of 2 signal periods. These results demonstrate that the 4.7-ps transformed-limited Gaussian pulses derived from stage 1 (Fig. 4(1), are efficiently reshaped into a linearly chirped parabolic shape after their successive propagations in the NZ-DSF and HNLF (Fig. 43). Final output pulses (Fig. 4(4) exhibit a nearly side-lobe free temporal intensity profile with a temporal width of $1.8 \mathrm{ps}$ (duty cycle of 1/14). The chirp is flat over most of the central part of the pulse. The spectrum of the generated 1.8-ps $40-\mathrm{GHz}$ pulse train is plotted in Fig. 3 and exhibits a symmetric good quality envelope constituted by many generated harmonics separated by $40 \mathrm{GHz}$ as well as an optical signal noise ratio in excess of $35 \mathrm{~dB}$. The generated pulses are then fully suitable for 160 $\mathrm{GHz}$ OTDM telecommunication applications.
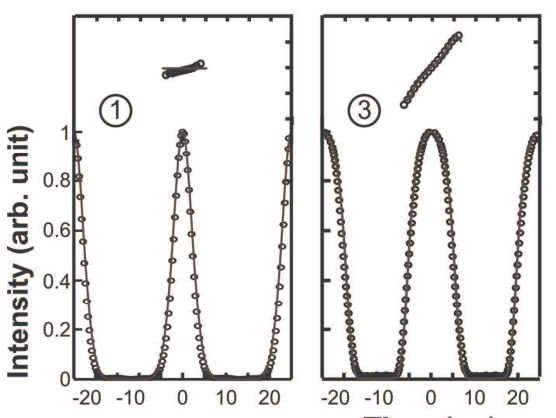

Time (ps)

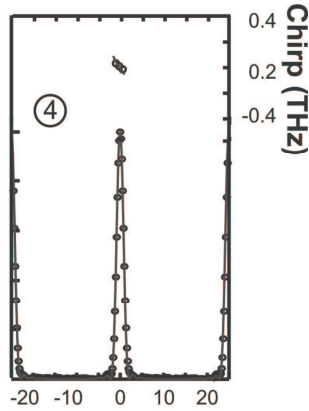

Fig. 4. Intensity and chirp profiles at stages 1,3 and 4. 40-GHz experimental results obtained by FROG characterisation (circles) are compared to numerical simulations of the Nonlinear Schrödinger Equation. (solid line).

Experimental results are finally compared with those obtained from the numerical integration of the standard Nonlinear Schrödinger Equation (Fig. 4, solid lines). The agreement is excellent and proves the reliability of the technique. We have also checked that higher-order linear and non-linear effects do not affect our proposed device at this repetition rate.

\section{CONCLUSION}

We have proposed a new and easy to implement method to generate carrier-suppressed pulse return-to-zero trains with low-duty-cycles. An initial sinusoidal beating is converted into nearly transformed limited pulses with very low substructures through the propagation in only four segments of optical fiber whose dispersion and length are carefully chosen. We have derived general guidelines which facilitate the choice and speed-up the scaling of the fibers and powers involved in the nonlinear processes. Experimental results obtained at $20 \mathrm{GHz}$ and $40 \mathrm{GHz}$ show that picosecond pulse trains with duty cycles as low as $1 / 14$ have been generated, thus validating this alternative method to comb-like dispersion decreasing techniques. By reducing the number of segments, we have also relaxed the constraint of tedious splices and consequent losses. A carefully characterisation both in intensity and phase fully agrees with numerical simulations and confirm our approach.

\section{ACKNOWLEDGMENT}

We would like to acknowledge OFS and more specifically L. Grüner-Nielsen for providing the highly nonlinear fiber. We also thank P. Petropoulos and D.J. Richardson (Optoelectronics Research Centre, UK), as well as V. Couderc (XLim, Limoges) for fruitful discussions.

\section{REFERENCES}

[1] P. V. Mamyshev, S. V. Chernikov, and E. M. Dianov, "Generation of fundamental soliton trains for high-bit rate optical fiber communication lines," IEEE J. Quantum Electron., vol. 27, pp. 2347-2355, 1991.

[2] S. V. Chernikov, J. R. Taylor, and R. Kashyap, "Comblike dispersionprofiled fiber for soliton pulse train generation," Opt. Lett., vol. 19, pp. 539-541, 1994

[3] K. Igarashi, H. Tobioka, M. Takahashi, T. Yagi, and S. Namiki, "Widely wavelength-tunable $40 \mathrm{GHz}$ femtosecond pulse source based on compression of externally-modulated pulse using $1.4 \mathrm{~km}$ comb-like profiled fibre," Electron. Lett., vol. 41, 2005.

[4] J. Fatome, S. Pitois, and G. Millot, "20-GHz-to-1-THz repetition rate pulse sources based on multiple four-wave mixing in optical fibers," IEEE J. Quantum Electron., vol. 42, pp. 1038-1046, 2006.

[5] S. Pitois, C. Finot, J. Fatome, and G. Millot, "Generation of 20-Ghz picosecond pulse trains in the normal and anomalous dispersion regimes of optical fibers," Opt. Commun., vol. 260, pp. 301-306, 2006.

[6] C. Finot, L. Provost, P. Petropoulos, and D. J. Richardson, "Parabolic pulse generation through passive nonlinear pulse reshaping in a normally dispersive two segment fiber device," Opt. Express, vol. 15, pp. 852-864, 2007.

[7] F. Parmigiani, C. Finot, K. Mukasa, M. Ibsen, M. A. F. Roelens, P. Petropoulos, and D. J. Richardson, "Ultra-flat SPM-broadened spectra in a highly nonlinear fiber using parabolic pulses formed in a fiber Bragg grating," Opt. Express, vol. 14, pp. 7617-7622, 2006.

[8] C. Finot, F. Parmigiani, P. Petropoulos, and D. J. Richardson, "Parabolic pulse evolution in normally dispersive fiber amplifiers preceding the similariton formation regime," Opt. Express, vol. 14, pp. 3161-3170, 2006.

[9] J. Fatome, S. Pitois, and G. Millot, "Sensitivity of SHG-FROG for the Characterisation of Ultrahigh-Repetition-Rate Telecommunication Laser Sources," Opt. Fiber. Technol., vol. 10, pp. 73-78, 2004. 